\title{
MINIREVIEW
}

\section{Animal models of maternal depression for monitoring neurodevelopmental changes occurring in dams and offspring}

\author{
Eszter BOGI 1, Kristína BELOVICOVA 1,2, Kristína CSATLOSOVA 1,2, Michal DUBOVICKY ${ }^{1}$ \\ ${ }^{1}$ Institute of Experimental Pharmacology and Toxicology, Slovak Academy of Sciences, Bratislava, Slovak Republic \\ 2 Department of Pharmacology, Jessenius Faculty of Medicine, Comenius University, Martin, Slovak Republic
}

ITX100117A05 • Received: 16 January 2017 • Accepted: 17 March 2017

\begin{abstract}
Depression is one of the most prevalent and life-threatening forms of mental illness affecting about 20\% of the population. Depressive disorder as a biochemical phenomenon, was first recognized in the mid-20th century of research, however the etiology of this disease is still not well understood. Although the need to investigate depressive disorders has emerged from the needs of clinical practice, there are many preclinical studies, which brought new insights into this field of research. During experimental work it was crucial to develop appropriate animal models, where the neurohumoral mechanism was similar to humans. In the past decades, several animal models of maternal depression have been developed. We describe the three most popular rodent models of maternal depression which are based on 1. stress prior to gestation, 2. prenatal stress and 3. early life stress. The above-mentioned animal models appear to fulfill many criteria for a relevant animal model of depression; they alter the regulation of the HPA, induce signs of depression-like behavior and several antidepressant treatments can reverse the state induced by maternal stress. Although, they are not able to model all aspects of maternal depression, they are useful models for monitoring neurodevelopmental changes occurring in dams and offspring.
\end{abstract}

KEY WORDS: rat; animal model; depression; pregnancy

\section{Introduction}

Depression is a mood disorder that causes a persistent feeling of sadness, loss of interest or pleasure in normal activities, sleep disturbances, tiredness, reduced appetite, feelings of worthlessness or suicidal thoughts (DSM-V). Depressive disorder as a biochemical phenomenon, was first recognized in the mid-20 ${ }^{\text {th }}$ century (Ashcroft et al., 1966; Schildkraut, 1965). Nowadays, depression is one of the most prevalent and life-threatening forms of mental illness affecting about $20 \%$ of the population (Pearlstein, 2015; Gentile, 2017). Despite half a century of research, the etiology of this disease is still not well understood and the treatment for depression also raises many questions. Currently, the symptoms of depressive disorders are related to monoamine depletion, excess of

\footnotetext{
Correspondence address:

Eszter Bogi, PhD.

Institute of Experimental Pharmacology and Toxicology,

Slovak Academy of Sciences,

Dúbravská cesta 9, 84104, Bratislava, Slovak Republic.

E-MAlL: eszter.bogi@savba.sk
}

corticotrophin-releasing hormone (CRH) and cortisol levels, glucocorticoid receptor (GR) resistance and modified cytokines levels. These alterations might be intimately involved in major mood changes.

Although the need to investigate depressive disorders has emerged from the needs of clinical practice, there are many preclinical studies, which brought new insights into this field of research. During experimental work it was crucial to develop suitable animal models, where the neurohumoral mechanism was similar to humans. However, designing a proper animal model of depression has some difficulties, since in animals we cannot detect signs of depressive disorder such as depressed mood, low selfconfidence, feeling of worthlessness or suicidal thoughts. On the other hand, there are some depression-associated endophenotypes that can be modelled in rodents. For example, anhedonia, which relates to loss of interest in pleasurable and/or rewarding actions. In rodents, anhedonia, can be assessed by the preference for a reward, such as sucrose solution (sucrose test) (Belzung \& Lemoine, 2011). Another manifestation can be behavioral despair of rats, which can be assessed with the forced swim test 
or with the tail suspension test (Belzung \& Lemoine, 2011; Wainwright \& Galea, 2013). Moreover, signs of depression both in humans and in rodents can be manifested by several other changes: 1. physiological (weight gain), 2. endocrinological (adrenocortical hormones) and 3. neuroanatomical (loss of neurons and dendritic atrophy in hippocampus), which can be reliably reproduced and evaluated in rodents (Deussing, 2006; Wainwright \& Galea, 2013).

In the past several animal models of depression have been developed. Most of the models were based on exposure to different types of excessive stress (Willner, 2005; Deussing, 2006; Mairesse et al., 2015). Stressful events have been shown to play an important role in the development and manifestation of psychiatric illnesses. When an organism is exposed to stress, catecholamines are released from the sympathetic nervous system. This release triggers secretion of $\mathrm{CRH}$ by neurosecretory cells in the hypothalamus. Subsequently CRH enhances the activation of adrenocorticotropic hormone (ACTH). Glucocorticoids are released from the adrenal gland in response to increased level of ACTH. This physiological stress response is a fundamental adaptation for an organism to cope with emergencies. However, when stress is excessive the mentioned alterations might lead to psychopathological disorders, such as depressive disorder (Korte, 2001). Chronic excessive stress results in glucocorticoid hypersecretion and can lead to long lasting hypothalamicpituitary-adrenal (HPA) responses. Furthermore, elevated levels of glucocorticoids inhibit HPA activity, which is associated with impaired glucocorticoid negative feedback in depressed patients. Indeed, depressed individuals have an abnormal HPA function which is manifested by hypersecretion of cortisol (Burke et al., 2005; Schüle, 2007; Brummelte \& Galea, 2010) and with abnormal cortisol rhythms, with lower cortisol levels in the morning and higher levels in the evening (Burke et al., 2005). While increased evening cortisol levels predict a greater risk for persistent depressive episodes (Goodyer et al., 2001). Moreover, elevation of cortisol level during chronic stress exerts neurotoxic effect on hippocampal neurons, which results in decreased neurogenesis, disrupted synaptogenesis, reduced dendritic spines. The morphological loss of neurons further leads to functional deficits, loss of long-term potentiation or long-term depression of hippocampus (Egeland et al., 2015). These neurochemical changes together determine the background of depressive disorders.

Similarly, it has been shown, that rats exposed chronically to a variety of unpredictable stressors showed sign of anhedonia and depressive-like behavior (Willner et al., 1987; Gross \& Pinhasov, 2016a). Animal studies have suggested that excessive stress is associated with higher corticosterone and aldosterone level (Jezova et al., 1996; Varga et al., 2013), impaired hippocampal neurogenesis and development of dendrites and synapses (Muhammad \& Kolb, 2011; Rayen et al., 2015), changes in social memory (Grundwald \& Brunton, 2015), alterations in circadian systems of the rats (Kiryanova et al., 2017) and with altered reactivity to pain or stress (Knaepen et al., 2014). Therefore, well-controlled animal studies could play an important role in the preclinical study of stressrelated disorders.

\section{Models of early life stress}

Women are more likely to have depression, especially with their first depressive episodes often occurring during pregnancy or lactation. Perinatal mood disorders occur in 10-15\% of pregnant women (Pearlstein, 2015; Kiryanova et al., 2017; van den Bergh et al., 2017). The stress experienced during pregnancy and lactation or even prior to gestation can negatively affect the mother and the offspring (Clements et al., 2015; Braun et al., 2017). Early adverse experiences have profound and long-lasting effects on the development of neurobiological systems. Stress during pregnancy leads to activation of the maternal, fetal and placental hypothalamic-pituitary-adrenal (HPA) axis, which stimulates a response contributing to maintain physiological conditions and at the same time avoiding the adverse effects of stress on the mother and the offspring. However, when this exceeds certain limits of intensity, a number of obstetric complications may occur (Voltolini \& Petraglia, 2014). The consequences of antenatal or postnatal depression during critical early brain development may lead to adverse effects on emotional, social and neurocognitive development in infants and increase vulnerability to psychiatric disorders in adulthood (Talge et al., 2007; Tarantino et al., 2011). Nevertheless, treatment during gestation and lactation raises also a number of questions, which are mainly related to the safety use of psychiatric drugs during this period. The main concern is, that antidepressants cross the placental and bloodbrain barrier and are excreted into the breastmilk, which increases the level of monoamines in the developing fetus. Since these neurotransmitters play an important role in the fetal maturation of the brain - during proliferation, myelinization and synaptogenesis, antidepressant therapy could interfere with these sensitive developmental processes in the brain and might have neurobehavioral consequences in later life. In clinical studies it is often difficult to separate consequences of the treatment alone and the effect of maternal depression. Preclinical work using appropriate animal models can give us answers about long-term consequences of prenatal stress and/or antidepressant therapy.

Although, rodent gestation is different from human pregnancy - neuroendocrinological changes during pregnancy and right after the birth are similar. However, from the developmental point of view it is important to note, that rodents are among the altricial (non-precocial) species, where an intensive maturation of the brain occurs in the postpartum period with a peak of around days 10-12 (Dobbing \& Sands, 1979). In humans, the intensive brain maturation culminates in the period around childbirth. Therefore, neurodevelopment in rats during pregnancy and in the first 10 postnatal days appears to correspond 
roughly to the third trimester of pregnancy and first month in humans neurodevelopment (Dobbing \& Sands, 1979; Rice et al., 2000). Consequently, rodent models could be useful tools for monitoring neurodevelopmental changes occurring in the prenatal period of humans.

In the past decades, several animal models of maternal depression have been developed. Models of maternal depression are based on prenatal and/or early life stress (Pereira-Figueiredo et al., 2017). Maternal depression models can be classified based on period (stress prior to gestation, prenatal stress and early life stress), stressor properties (e.g., physical or social) and duration (acute or chronic) of the exposure (Stepanichev et al., 2014). We describe the three most popular rodent models of maternal depression which are based on 1. stress prior to gestation, 2. prenatal stress and 3. early life stress. The diversity of approaches, which are used to investigate maternal depression in animal models contribute to the variety of the observed changes in the prenatally stressed offspring.

\section{Model of chronic unpredictable stress CUS}

This model study was designed to clarify whether pregestational stress affects offspring in later life. Non-gravid females are exposed to 3-weeks of stress in the form of different stressors, which are changed every day at different points in time (Willner et al., 1987; Willner \& Mitchell, 2002; Willner, 2005; Xing et al., 2013). Pre-gestational stress caused neuroendocrinological changes in rat dams and their offspring by a significant increase in $\mathrm{CRH}$ and corticosterone via maternal, fetal, and placental HPA axis (Huang et al., 2012). These early neurochemical changes might alter the entire programing of the hypothalamicpituitary-adrenal (HPA) axis function. Furthermore, alterations in HPA axis activity could be related to functional changes in serotonin activity and serotonin transporter expression in the hippocampus and hypothalamus of fetuses in a sexually dimorphic way (Huang et al., 2012, 2016). Pre-gestational stress also appears to cause long-lasting changes in hippocampal mechanisms in the offspring rats (Huang et al., 2013; Gemmel et al., 2017). Moreover, changes in HPA axis are linked to alterations in depressive-like and social behavior. Offspring of affected mothers showed decreased coping ability in the forced swimming and deficits in sibling play behavior of juvenile offspring (Grundwald et al., 2016; Gemmel et al., 2017). All these neurochemical, neurobehavioral changes could result in the decreased ability of an organism to respond and adapt to stressful stimuli later in adolescent or in adulthood (Huang et al., 2016). At the same time, perinatal antidepressant exposure had an enduring effect on the HPA system and the central serotonergic system by increasing serotonin and CBG levels in the hippocampus (Gemmel et al., 2017).

\section{Restraint stress}

Restraint stress involves exposure of pregnant dams to restraint stress by placing them in restraint tubes for a period of time over several days. Dams undergoing this model, have shown increased depression-like behavior (Mairesse et al., 2015). The offspring of affected mothers exhibit increased ultrasonic vocalization in juvenile age, reduced social play during adolescence and increased immobility time in the forced swim test during adulthood and other behavioral changes including disturbed circadian rhythms, altered HPA axis feedback mechanisms and neuroplasticity (Maccari \& Morley-Fletcher, 2007; Mairesse et al., 2015). Moreover, adult offspring of affected mothers showed a reduced density of benzodiazepine recognition sites at GABA receptors in the hippocampus and in amygdala (Barros et al., 2006), as well as reduced hippocampal neuroplasticity (Zuena et al., 2008). Early postnatal exposure to maternal fluoxetine reversed the decrease in immobility in the FST, hippocampal cell proliferation and hippocampal neurogenesis in maternally stressed adolescent offspring (Rayen et al., 2011), however, this preventative effect of SSRIs against the effects of maternal stress is not evident during adulthood (Rayen et al., 2015).

\section{Maternal separation}

This early life stress model is based on the paradigm that stress during critical periods in development can have severe long-term consequences in later life. During separation sessions, pups are removed from their mother. Multiple variations of the maternal deprivation procedure have been developed, with different length of the separation episodes (1-24h in 1-14 days). While the time frame and length of mother segregation correlates with the severity of adolescence and adulthood disorders (Ladd et al., 1996), maternal separation leads to an increased anxiety- and depression-like behavior during adolescent and adulthood (Finamore \& Port., 2000; Gonzalez et al., 2001) and to changes in the HPA axis in terms of elevated corticosterone level (Ladd et al., 1996; Ladd et al., 2000). Furthermore, excessive glucocorticoid activity in this model has been shown to decrease hippocampal neurogenesis (Bredy et al., 2003). Brain monoamine systems also appear to be altered by maternal separation. Especially, rats showed increased CRF expression in locus coeruleus and raphe nuclei (Plotsky \& Meaney, 1993). Maternal separation can cause various endocrinological and behavioral changes manifested in later life, which could be caused by alterations in HPA system and through epigenetic mechanisms.

\section{Conclusion}

The above-mentioned animal models appear to fulfill many criteria for a relevant animal model of depression; they alter the regulation of the HPA, induce signs of depression-like behavior and several antidepressant treatments can reverse the state induced by maternal stress. Although, they are not able to model all aspects of maternal depression, they are useful models for monitoring neurodevelopmental changes occurring in dams and offspring. 


\section{Acknowledgement}

This study was supported by the Slovak Research Agency by realization of the project: „Transfer of Knowledge and Technologies from Research and Development in Toxicology on Evaluation of Environmental and Health Risks “(ITMS 26240220005).

\section{REFERENCES}

Ashcroft GW, Crawford TB, Eccleston D, Sharman DF, MacDougall EJ, Stanton JB, Binns JK. (1966). 5-hydroxyindole compounds in the cerebrospinal fluid of patients with psychiatric or neurological diseases. Lancet (London, England) 2: 1049-52.

Barros VG, Rodríguez P, Martijena ID, Pérez A, Molina VA, Antonelli MC. (2006). Prenatal stress and early adoption effects on benzodiazepine receptors and anxiogenic behavior in the adult rat brain. Synapse 60: 609-18.

Belzung C, Lemoine M. (2011). Criteria of validity for animal models of psychiatric disorders: focus on anxiety disorders and depression. Biol. Mood Anxiety Disord. 1: 9 .

Braun K, Bock J, Wainstock T, Matas E, Gaisler-Salomon I, Fegert J, Ziegenhain U, Segal M. (2017). Experience-induced transgenerational (re-)programming of neuronal structure and functions: Impact of stress prior and during pregnancy. Neurosci Biobehav Rev. pii: S0149-7634(16)30731-X. [Epub ahead of print

Bredy TW, Grant RJ, Champagne DL, Meaney MJ. (2003). Maternal care influences neuronal survival in the hippocampus of the rat. Eur. J. Neurosci. 18: 2903-9.

Brummelte S, Galea LAM. (2010). Depression during pregnancy and postpartum: contribution of stress and ovarian hormones. Prog. Neuropsychopharmacol. Biol. Psychiatry 34: 766-76.

Burke HM, Davis MC, Otte C, Mohr DC. (2005). Depression and cortisol responses to psychological stress: a meta-analysis. Psychoneuroendocrinology 30: 846-56.

Clements CC, Castro VM, Blumenthal SR, Rosenfield HR, Murphy SN, Fava M, Erb JL, Churchill SE, Kaimal AJ, Doyle AE, Robinson EB, Smoller JW, Kohane IS, Perlis RH. (2015). Prenatal antidepressant exposure is associated with risk for attention-deficit hyperactivity disorder but not autism spectrum disorder in a large health system. Mol. Psychiatry 20: 727-34.

Deussing JM. (2006). Animal models of depression. Drug Discov. Today Dis. Model. 3: 375-383.

Dobbing J, Sands J. (1979). Comparative aspects of the brain growth spurt. Early Hum. Dev. 3: 79-83.

Egeland M, Zunszain PA, Pariante CM. (2015). Molecular mechanisms in the regulation of adult neurogenesis during stress. Nat. Rev. Neurosci. 16: 189200

Finamore TL, Port RL. Developmental stress disrupts habituation but spares prepulse inhibition in young rats. Physiol. Behav. 69: 527-30.

Gemmel M, Hazlett M, Bögi E, De Lacalle S, Hill LA, Kokras N, Hammond GL, Dalla C, Charlier TD, Pawluski JL. (2017). Perinatal fluoxetine effects on social play, the HPA system, and hippocampal plasticity in pre-adolescent male and female rats: Interactions with pre-gestational maternal stress. Psychoneuroendocrinology 84: 159-171.

Gentile S. (2011). Suicidal mothers. J. Inj. Violence Res. 3: 90-7.

Gentile S. (2017). Untreated depression during pregnancy: Short- and longterm effects in offspring. A systematic review. Neuroscience 342: 154-166.

Gonzalez A, Lovic V, Ward GR, Wainwright PE, Fleming AS. (2001). Intergenerational effects of complete maternal deprivation and replacement stimulation on maternal behavior and emotionality in female rats. Dev. Psychobiol. 38: 11-32.

Goodyer IM, Park RJ, Herbert J. (2001). Psychosocial and endocrine features of chronic first-episode major depression in 8-16 year olds. Biol. Psychiatry 50: 351-7.

Gross M, Pinhasov A. (2016). Chronic mild stress in submissive mice: Marked polydipsia and social avoidance without hedonic deficit in the sucrose preference test. Behav. Brain Res. 298: 25-34.
Grundwald NJ, Benítez DP, Brunton PJ. (2016). Sex-Dependent Effects of Prenatal Stress on Social Memory in Rats: A Role for Differential Expression of Central Vasopressin-1a Receptors. J. Neuroendocrinol. 28(4). doi: 10.1111/ jne.12343.

Grundwald NJ, Brunton PJ. (2015). Prenatal stress programs neuroendocrine stress responses and affective behaviors in second generation rats in a sexdependent manner. Psychoneuroendocrinology 62: 204-216.

Huang Y, Chen S, Xu H, Yu X, Lai H, Ho G, Huang Q, Shi X. (2013). Pre-gestational stress alters stress-response of pubertal offspring rat in sexually dimorphic and hemispherically asymmetric manner. BMC Neurosci. 14:67.

Huang Y, Shen Z, Hu L, Xia F, Li Y, Zhuang J, Chen P, Huang Q. (2016). Exposure of mother rats to chronic unpredictable stress before pregnancy alters the metabolism of gamma-aminobutyric acid and glutamate in the right hippocampus of offspring in early adolescence in a sexually dimorphic manner. Psychiatry Res. 246: 236-245.

Huang Y, Xu H, Li H, Yang H, Chen Y, Shi X. (2012). Pre-gestational stress reduces the ratio of 5-HIAA to 5-HT and the expression of 5-HT1A receptor and serotonin transporter in the brain of foetal rat. BMC Neurosci. 13: 22.

Jezová D, Juránková E, Mosnárová A, Kriska M, Skultétyová I. (1996). Neuroendocrine response during stress with relation to gender differences. Acta Neurobiol. Exp. (Wars). 56: 779-85.

Kiryanova V, Smith VM, Dyck RH, Antle MC. (2017a). Circadian behavior of adult mice exposed to stress and fluoxetine during development. Psychopharmacology (Berl). 234: 793-804.

Knaepen L, Pawluski JL, Patijn J, van Kleef M, Tibboel D, Joosten EA. (2014). Perinatal maternal stress and serotonin signaling: Effects on pain sensitivity in offspring. Dev. Psychobiol. 56: 885-896.

Korte S. (2001). Corticosteroids in relation to fear, anxiety and psychopathology. Neurosci. Biobehav. Rev. 25: 117-142.

Ladd CO, Huot RL, Thrivikraman K V, Nemeroff CB, Meaney MJ, Plotsky PM. (2000). Long-term behavioral and neuroendocrine adaptations to adverse early experience. Prog. Brain Res. 122: 81-103.

Ladd CO, Owens MJ, Nemeroff CB. (1996). Persistent changes in corticotropin-releasing factor neuronal systems induced by maternal deprivation. Endocrinology 137: 1212-8.

Maccari S, Morley-Fletcher S. (2007). Effects of prenatal restraint stress on the hypothalamus-pituitary-adrenal axis and related behavioural and neurobiological alterations. Psychoneuroendocrinology 32 Suppl 1: S10-5.

Mairesse J, Van Camp G, Gatta E, Marrocco J, Reynaert M-L, Consolazione M, Morley-Fletcher S, Nicoletti F, Maccari S. (2015). Sleep in prenatally restraint stressed rats, a model of mixed anxiety-depressive disorder. Adv. Neurobiol. 10: 27-44.

Muhammad A, Kolb B. (2011). Mild prenatal stress-modulated behavior and neuronal spine density without affecting amphetamine sensitization. Dev. Neurosci. 33: 85-98.

Pearlstein T. (2015). Depression during Pregnancy. Best Pract. Res. Clin. Obstet. Gynaecol. 29: 754-64.

Pereira-Figueiredo I, Castellano O, Riolobos AS, Ferreira-Dias G, López DE, Sancho C. (2017). Long-Term Sertraline Intake Reverses the Behavioral Changes Induced by Prenatal Stress in Rats in a Sex-Dependent Way. Front. Behav. Neurosci. 11: 99.

Plotsky PM, Meaney MJ. (1993). Early, postnatal experience alters hypothalamic corticotropin-releasing factor (CRF) mRNA, median eminence CRF content and stress-induced release in adult rats. Brain Res. Mol. Brain Res. 18: $195-200$.

Rayen I, Gemmel M, Pauley G, Steinbusch HWM, Pawluski JL. (2015). Developmental exposure to SSRIs, in addition to maternal stress, has long-term sex-dependent effects on hippocampal plasticity. Psychopharmacology (Berl). 232: 1231-1244.

Rayen I, van den Hove DL, Prickaerts J, Steinbusch HW, Pawluski JL. (2011). Fluoxetine during development reverses the effects of prenatal stress on depressive-like behavior and hippocampal neurogenesis in adolescence. PLoS One 6: e24003.

Rice D, Barone S, Jr. (2000). Critical periods of vulnerability for the developing nervous system: evidence from humans and animal models. Environ. Health Perspect. 108 Suppl 3: 511-33.

Schildkraut JJ. (1965). The catecholamine hypothesis of affective disorders: a review of supporting evidence. Am J Psychiatry. 122(5): 509-22.

Schüle C. (2007). Neuroendocrinological mechanisms of actions of antidepressant drugs. J. Neuroendocrinol. 19: 213-26. 
Stepanichev M, Dygalo NN, Grigoryan G, Shishkina GT, Gulyaeva N. (2014). Rodent models of depression: neurotrophic and neuroinflammatory biomarkers. Biomed Res. Int. 2014: 932757.

Talge NM, Neal C, Glover V. (2007). Antenatal maternal stress and long-term effects on child neurodevelopment: how and why? J. Child Psychol. Psychiatry. 48(3-4): 245-61.

Tarantino LM, Sullivan PF, Meltzer-Brody S. (2011). Using animal models to disentangle the role of genetic, epigenetic, and environmental influences on behavioral outcomes associated with maternal anxiety and depression. Front. psychiatry 2: 44.

van den Bergh BRH, van den Heuvel MI, Lahti M, Braeken M, de Rooij SR, Entringer S, Hoyer D, Roseboom T, Räikkönen K, King S, Schwab M. (2017) Prenatal developmental origins of behavior and mental health: the influence of maternal stress in pregnancy. Neurosci. Biobehav. Rev. pii: S01497634(16)30734-5, [Epub ahead of print]

Varga J, Ferenczi S, Kovács KJ, Garafova A, Jezova D, Zelena D. (2013). Comparison of stress-induced changes in adults and pups: is aldosterone the main adrenocortical stress hormone during the perinatal period in rats? PLoS One 8: e72313.

Voltolini C, Petraglia F. (2014). Neuroendocrinology of pregnancy and parturition. Handb Clin Neurol. 124: 17-36.
Wainwright SR, Galea LAM. (2013). The neural plasticity theory of depression: assessing the roles of adult neurogenesis and PSA-NCAM within the hippocampus. Neural Plast. 2013: 805497.

Willner P. (2005). Chronic mild stress (CMS) revisited: consistency and behavioural-neurobiological concordance in the effects of CMS. Neuropsychobiology 52: 90-110.

Willner P, Mitchell PJ. (2002). The validity of animal models of predisposition to depression. Behav. Pharmacol. 13: 169-88.

Willner P, Towell A, Sampson D, Sophokleous S, Muscat R. (1987). Reduction of sucrose preference by chronic unpredictable mild stress, and its restoration by a tricyclic antidepressant. Psychopharmacology (Berl). 93: 358-64.

Xing Y, He J, Hou J, Lin F, Tian J, Kurihara H. (2013). Gender differences in CMS and the effects of antidepressant venlafaxine in rats. Neurochem. Int. 63: 570-5.

Zuena AR, Mairesse J, Casolini P, Cinque C, Alemà GS, Morley-Fletcher S, Chiodi V, Spagnoli LG, Gradini R, Catalani A, Nicoletti F, Maccari S. (2008). Prenatal restraint stress generates two distinct behavioral and neurochemical profiles in male and female rats. PLoS One 3: e2170. 